\title{
Simultaneous determination of production and shipment decisions for a multi-product inventory system with a rework process
}

\author{
Chiu, Y.P. ${ }^{a}$, Chiang, K.-W. ${ }^{a}$, Chiu, S.W. ${ }^{\text {b, }}$, Song, M.-S. ${ }^{a}$ \\ ${ }^{a}$ Department of Industrial Engineering and Management, Chaoyang University of Technology, Wufong, Taichung, \\ Taiwan, R.O.C. \\ ${ }^{b}$ Department of Business Administration, Chaoyang University of Technology, Wufong, Taichung, Taiwan, R.O.C.
}

\section{A B S T R A C T}

In a turbulent and highly competitive business environment, management always pursues options to reduce overall operating costs. The vendor-buyer integrated system has recently drawn attention from managers, because it can benefit both parties of the supply chain and it is suitable to be applied to a so-called intra-supply chain system within the present-day globalized enterprise. This study attempts to simultaneously determine production and shipment decisions for a multi-product vendor-buyer integrated inventory system with a rework process, wherein multiple products are fabricated in sequence by a single machine under a rotation cycle time policy. All defective items produced in regular production are assumed repairable, and are reworked right after the regular production ends. Finished goods of each product are transported to sales offices/customers after rework. A multi-delivery policy is applied, wherein a fixed quantity of $n$ instalments of the finished batch is delivered at fixed intervals during the delivery timeframe. Mathematical modelling and optimization techniques are used to help simultaneously determine the optimal production and shipment decisions that minimize the expected overall system costs. A numerical example is used to show the applicability of our research results.
\end{abstract}

\section{ARTICLE INFO}

Keywords:

Multi-product inventory system Vendor-buyer integrated system Intra-supply chain Common production cycle time Rework

*Corresponding author: swang@cyut.edu.tw (Chiu, S.W.)

Article history:

Received 31 January 2016

Revised 20 May 2016

Accepted 22 May 2016

\section{References}

[1] Tersine, R.J. (1994). Principles of inventory and materials management, PTR Prentice-Hall, New Jersey, USA.

[2] Zipkin, P.H. (2000). Foundations of inventory management, McGraw-Hill, New York, USA

[3] Nahmias, S. (2009). Production and operations analysis, (6 ${ }^{\text {th }}$ edition), McGraw-Hill, New York, USA.

[4] Gaalman, G.J. (1978). Optimal aggregation of multi-item production smoothing models, Management Science, Vol. 24, No. 16, 1733-1739, doi: 10.1287/mnsc.24.16.1733.

[5] Leachman, R.C., Gascon, A. (1988). A heuristic scheduling policy for multi-item, single-machine production systems with time-varying, stochastic demands, Management Science, Vol. 34, No. 3, 377-390, doi: 10.1287/ mnsc.34.3.377.

[6] Zipkin, P.H. (1995). Performance analysis of a multi-item production-inventory system under alternative policies, Management Science, Vol. 41, No. 4, 690-703, doi: 10.1287/mnsc.41.4.690.

[7] Muramatsu, K., Warman, A., Kobayashi, M. (2003). A near-optimal solution method of multi-item multi-process dynamic lot size scheduling problem, JSME International Journal, Series C: Mechanical Systems, Machine Elements and Manufacturing, Vol. 46, No. 1, 46-53, doi: 10.1299/jsmec.46.46.

[8] Jodlbauer, H., Reitner, S. (2012). Optimizing service-level and relevant cost for a stochastic multi-item cyclic production system, International Journal of Production Economics, Vol. 136, No. 2, 306-317, doi: 10.1016/ j.ijpe.2011.12.015. 
[9] Gordon, G.R., Surkis, J. (1975). A control policy for multi-item inventories with fluctuating demand using simulation, Computers \& Operations Research, Vol. 2, No. 2, 91-100, doi: 10.1016/0305-0548(75)90012-X.

[10] Aggarwal, V. (1984). Grouping multi-item inventory using common cycle periods, European Journal of Operational Research, Vol. 17, No. 3, 369-372, doi: 10.1016/0377-2217(84)90132-2.

[11] Federgruen, A., Katalan, Z. (1998). Determining production schedules under base-stock policies in single facility multi-item production systems, Operations Research, Vol. 46, No. 6, 883-898, doi: 10.1287/opre.46.6.883.

[12] Guchhait, P., Maiti, M.K., Maiti, M. (2010). Multi-item inventory model of breakable items with stock-dependent demand under stock and time dependent breakability rate, Computers \& Industrial Engineering, Vol. 59, No. 4, 911-920, doi: 10.1016/i.cie.2010.09.001.

[13] Chiu, Y.-S.P., Huang, C.-C., Wu, M.-F., Chang, H.-H. (2013). Joint determination of rotation cycle time and number of shipments for a multi-item EPQ model with random defective rate, Economic Modelling, Vol. 35, 112-117, doi: 10.1016/i.econmod.2013.06.024.

[14] Wu, M.-F., Chiu, Y.-S.P., Sung, P.-C. (2014). Optimization of a multi-product EPQ model with scrap and an improved multi-delivery policy, Journal of Engineering Research, Vol. 2, No. 4., 103-118, doi: 10.7603/s40632-0140027-7.

[15] Chiu, Y.-S.P., Sung, P.-C., Chiu, S.W., Chou, C.-L. (2015). Mathematical modeling of a multi-product EMQ model with an enhanced end items issuing policy and failures in rework, SpringerPlus, Vol. 4:679, No. 1, 1-11, doi: 10.1186/s40064-015-1487-4.

[16] Taft, E.W. (1918). The most economical production lot, Iron Age, Vol. 101, 1410-1412.

[17] Hahm, J., Yano, C.A. (1992). The economic lot and delivery scheduling problem: The single item case, International Journal of Production Economics, Vol. 28, No. 2, 235-252, doi: 10.1016/0925-5273(92)90036-7.

[18] Eben-Chaime, M. (2004). The effect of discreteness in vendor-buyer relationships, IIE Transactions, Vol. 36, No. 6, 583-589, doi: 10.1080/07408170490438717.

[19] Sarmah, S.P., Acharya, D., Goyal, S.K. (2008). Coordination of a single-manufacturer/multi-buyer supply chain with credit option, International Journal of Production Economics, Vol. 111, No. 2, 676-685, doi: 10.1016/ j.ijpe.2007.04.003.

[20] Schwarz, L.B., Deuermeyer, B.L., Badinelli, R.D. (1985). Fill-rate optimization in a one-warehouse N-identical retailer distribution system, Management Science, Vol. 31, No. 4, 488-498, doi: 10.1287/mnsc.31.4.488.

[21] Hall, R.W. (1996). On the integration of production and distribution: economic order and production quantity implications, Transportation Research Part B: Methodological, Vol. 30, No. 5, 387-403, doi: 10.1016/01912615(96)00002-1.

[22] Sarker, R.A., Khan, L.R. (1999). An optimal batch size for a production system operating under periodic delivery policy, Computers \& Industrial Engineering, Vol. 37, No. 4, 711-730, doi: 10.1016/S0360-8352(00)00006-1.

[23] Çömez, N., Stecke, K.E., Çakanyıldırım, M. (2012). Multiple in-cycle transshipments with positive delivery times, Production and Operations Management, Vol. 21, No. 2, 378-395, doi: 10.1111/i.1937-5956.2011.01244.X.

[24] Chiu, S.W., Sung, P.-C., Tseng, C.-T., Chiu, Y.-S.P. (2015). Multi-product FPR model with rework and multi-shipment policy resolved by algebraic approach, Journal of Scientific and Industrial Research, Vol. 74, No. 10, 555-559.

[25] Safaei, M. (2014). An integrated multi-objective model for allocating the limited sources in a multiple multi-stage lean supply chain, Economic Modelling, Vol. 37, 224-237, doi: 10.1016/j.econmod.2013.10.018.

[26] Ocampo, L.A. (2015). A hierarchical framework for index computation in sustainable manufacturing, Advances in Production Engineering \& Management, Vol. 10, No. 1, 40-50, doi: 10.14743/apem2015.1.191.

[27] Chiu, S.W., Huang, C.-C., Chiang, K.-W., Wu, M.-F. (2015). On intra-supply chain system with an improved distribution plan, multiple sales locations and quality assurance, SpringerPlus, Vol. 4:687, No. 1, 1-11, doi: 10.1186/ s40064-015-1498-1.

[28] Silver, E.A., Pyke, D.F., Peterson, R. (1998). Inventory management and production planning and scheduling, (3 3 rd edition), John Wiley \& Sons, New York, USA.

[29] Zargar, A.M. (1995). Effect of rework strategies on cycle time, Computers \& Industrial Engineering, Vol. 29, No. 14, 239-243, doi: 10.1016/0360-8352(95)00078-F.

[30] Inderfurth, K., Janiak, A., Kovalyov, M.Y., Werner, F. (2006). Batching work and rework processes with limited deterioration of reworkables, Computers \& Operations Research, Vol. 33, No. 6, 1595-1605, doi: 10.1016/i.cor. 2004.11.009.

[31] Chiu, Y-S.P., Pan, N., Chiu, S.W., Chiang, K.-W. (2012). Optimal production cycle time for multi-item FPR model with rework and multi-shipment policy, International Journal for Engineering Modelling, Vol. 25, No. 1-4, 51-57.

[32] Chiu, S.W., Lee, C.-H., Chiu, Y.-S.P., Cheng, F.-T. (2013). Intra-supply chain system with multiple sales locations and quality assurance, Expert Systems with Applications, Vol. 40, No. 7, 2669-2676, doi: 10.1016/j.eswa. 2012.11.008.

[33] Yu, K.-Y.C., Bricker, D.L. (1993). Analysis of a Markov chain model of a multistage manufacturing system with inspection, rejection, and rework, IIE Transactions, Vol. 25, No. 1, 109-112, doi: 10.1080/07408179308964271.

[34] Biswas, P., Sarker, B.R. (2008). Optimal batch quantity models for a lean production system with in-cycle rework and scrap, International Journal of Production Research, Vol. 46, No. 23, 6585-6610, doi: 10.1080/00207540 $\underline{802230330 .}$.

[35] Widyadana, G.A., Wee, H.M. (2011). Optimal deteriorating items production inventory models with random machine breakdown and stochastic repair time, Applied Mathematical Modelling, Vol. 35, No. 7, 3495-3508, doi: 10.1016/j.apm.2011.01.006.

[36] Chiu, Y.-S.P., Chen, Y.-C., Lin, H.-D., Chang, H.-H. (2014). Combining an improved multi-delivery policy into a single-producer multi-retailer integrated inventory system with scrap in production, Economic Modelling, Vol. 39, 163-167, doi: 10.1016/j.econmod.2014.02.031. 
[37] Tseng, C.-T., Wu, M.-F., Lin, H.-D., Chiu, Y.-S.P. (2014). Solving a vendor-buyer integrated problem with rework and a specific multi-delivery policy by a two-phase algebraic approach, Economic Modelling, Vol. 36, 30-36, doi: 10.1016/j.econmod.2013.09.013.

[38] Lin, G.C., Gong, D.-C., Chang, C.-C. (2014). On an economic production quantity model with two unreliable key components subject to random failures, Journal of Scientific and Industrial Research, Vol. 73, 149-152.

[39] Chiu, Y-S.P., Chang, H.-H. (2014). Optimal run time for EPQ model with scrap, rework and stochastic breakdowns: A note, Economic Modelling, Vol. 37, 143-148, doi: 10.1016/j.econmod.2013.11.006.

[40] Haider, A., Mirza, J. (2015). An implementation of lean scheduling in a job shop environment, Advances in Production Engineering \& Management, Vol. 10, No. 1, 5-17, doi: 10.14743/apem2015.1.188.

[41] Rardin, R.L. (1998). Optimization in operations research, Prentice-Hall, New Jersey, USA. 


\title{
Simultano določanje proizvodnje in odpreme za večizdelčni sistem zalog z možnostjo dodelav
}

\author{
Chiu, Y.P. ${ }^{a}$, Chiang, K.-W. ${ }^{a}$, Chiu, S.W..$^{\text {, }}{ }^{,}$, Song, M.-S. ${ }^{a}$ \\ ${ }^{a}$ Department of Industrial Engineering and Management, Chaoyang University of Technology, Wufong, Taichung, \\ Taiwan, R.O.C. \\ ${ }^{b}$ Department of Business Administration, Chaoyang University of Technology, Wufong, Taichung, Taiwan, R.O.C.
}

\begin{abstract}
POVZETEK
V turbolentnih in visoko konkurenčnih poslovnih okoljih menedžment vselej stremi k zniževanju operativnih stroškov. V zadnjem času menedžerji še posebno pozornost namenjajo integriranemu sistemu dobavitelj-kupec, saj koristi obema stranema v oskrbovalni verigi. Namen prispevka je simultano določanje proizvodnje in odpreme za večizdelčni sistem zalog dobaviteljkupec z dodelavami, kjer se več izdelkov izdeluje v zaporedju na enem stroju z rotirajočim ciklom. Pomanjkljivi kosi v rednem proizvodnem procesu se upoštevajo kot popravljivi in se dodelajo po končanju redne proizvodnje. $V$ delu sta uporabljeni matematično modeliranje in optimizacija za pomoč pri simultanemu odločanju za optimalne odločitve pri proizvodnji in pri odpremi, kar pripomore k nižjim stroškom delovanja sistema. Podan je številčen primer, ki nakazuje uporabnost naših raziskovalnih rezultatov.
\end{abstract}

(C) 2016 PEI, University of Maribor. All rights reserved.
PODATKI O ČLANKU

Ključne besede:

Večizdelčni sistem zalog

Integrirani sistem dobavitelj-kupec

Notranja oskrbovalna veriga

Skupni proizvodni cikel

Dodelava

*Kontaktna oseba:

swang@cyut.edu.tw

(Chiu, S.W.)

Zgodovina članka:

Prejet 31. januarja 2016

Popravljen 20. maja 2016

Sprejet 22. maja 2016 